Published in final edited form as:

Ann N Y Acad Sci. 2009 July ; 1170: . doi:10.1111/j.1749-6632.2009.04595.x.

\title{
Symposium Overview:
}

Sweet Taste: Receptors, Transduction, and Hormonal Modulation

\author{
Nirupa Chaudhari ${ }^{\mathrm{a}}$ and Sue C. Kinnamon ${ }^{\mathrm{b}, \mathrm{c}}$ \\ aDepartment of Physiology and Biophysics and Program in Neurosciences, University of Miami \\ Miller School of Medicine, Miami, Florida, USA \\ bDepartment of Biomedical Sciences, Colorado State University, Ft. Collins, Colorado, USA \\ ${ }^{c}$ Rocky Mountain Taste and Smell Center, Aurora, Colorado, USA
}

\begin{abstract}
In the last decade, a great deal of progress has been made in understanding the molecular and physiological basis of sweet taste. A heterodimeric G protein-coupled receptor, T1R2/ T1R3, appears to bind to and be activated by most (but not all) sweet tastants. ${ }^{1-4}$ The component subunit T1R3 was originally cloned based on its association with Sac, a murine locus that determines sensitivity and preference for sugars and synthetic sweeteners. 5,6 Structure-function studies on the sweet taste receptor expressed in cell culture revealed that each of the two subunits has binding sites for different sweeteners and plays a role in signaling to downstream partners. ${ }^{7-9}$ Activation of sweet receptors triggers a phosphoinositide and calcium signaling pathway that is common to all the taste G proteincoupled receptors for sweet, bitter, and umami ${ }^{10}$ and includes $\mathrm{G} \beta \gamma$ subunits, phospholipase $\mathrm{C} \beta 2$, and the nonselective cation channel, TrpM5.
\end{abstract}

Now that the principles of transduction for sweet taste have been outlined, the field has turned to examining the mechanisms that modulate sweet taste sensitivity and preference. The interaction of taste with physiological states in animals and people, and how this influences feeding and appetite, is of particular interest. Four speakers in this symposium highlighted interesting new developments that suggest that sweet taste may be particularly amenable to perturbation by hormonal and environmental influences.

Several peptide hormones serve as adiposity and satiety signals from peripheral tissues and affect hypothalamic and brain stem centers for appetite regulation. In recently published work, the laboratories of Steve Munger and Josephine Egan showed that one of these hormones, glucagon-like peptide 1 , in addition to its prominent role in the gut, appears also to be secreted from taste buds and to act on nearby nerve fibers. ${ }^{11}$ In his presentation, Steve Munger further reported on studies from his laboratory ${ }^{12}$ that suggest that glucagon-like peptide 1 modulates sweet and umami tastes in opposing directions. The implications for paracrine modulation in the taste periphery and/or for new avenues of neural signaling are substantial. Another peptide hormone, leptin, is synthesized in adipose tissue and has received much attention in the scientific and popular literature. Yuzo Ninomiya's group showed some years ago ${ }^{13,14}$ that leptin receptors expressed on mouse taste buds are activated by circulating leptin and serve to inhibit responses to sweet tastants. In this

(C) 2009 New York Academy of Sciences.

Address for correspondence: Dr. Nirupa Chaudhari, Dept. of Physiology and Biophysics, University of Miami Miller School of Medicine, 1600 NW 10th Ave., Miami, FL 33136. nchaudhari@miami.edu.

Conflicts of Interest

The authors declare no conflicts of interest. 
symposium, Ninomiya ${ }^{15}$ discussed recent experiments that suggest parallel effects may occur in humans as well.

Alan Carlton presented a summary of his recently published findings utilizing intrinsic imaging to map the spatial representation of taste qualities in the rat primary gustatory cortex. His data showed that stimuli representing sweet, salty, sour, and bitter taste qualities mapped to distinct, but overlapping, areas of the gustatory cortex. Stimuli that were of similar hedonic qualities (e.g., sweet and salty) had more overlapping distributions than stimuli of different hedonic qualities (e.g., sweet and bitter). ${ }^{16}$ Further, he presented data suggesting that these gustatory maps were plastic and could be modified by gustatory experience. Rats conditioned to avoid sucrose exhibited a map more typical of a bitter representation than a sweet one. However, after extinction of the conditioned taste aversion, the maps representing sweet and bitter became more spatially distinct, suggesting that the stimuli once again had different meanings. ${ }^{17}$ Taken together, these two studies suggest that the gustatory cortex encodes not only stimulus quality but also the hedonic value of a stimulus.

Gestational diabetes mellitus (GDM) is glucose intolerance first identified during pregnancy and affects as many as $9 \%$ of pregnant women. People with type 2 diabetes are known to exhibit an increased desire for sweet taste in their diet. Lisa Belzer reported on new studies that have examined the extent and time course for sweet taste changes in women who develop gestational diabetes and relate these alterations to the endocrine and metabolic profiles of pregnancy. Women with GDM showed higher preferences for sucrose-sweetened milk late in pregnancy, and a subset of these women reported more frequent sweet cravings than women with normal glucose tolerance. Moreover, in comparison to pregnant women with normal glucose tolerance, circulating insulin and leptin rose earlier in women who developed GDM, coinciding with the emergence of diabetic symptoms at midpregnancy. These premature elevations in insulin and leptin were positively correlated with higher liking ratings for sweet drinks in women with GDM. Thus, GDM may increase the hedonic value of sweet taste and cravings for sweet foods. Since diet is the cornerstone of GDM management, this work may lead to the development of better dietary management or prevention strategies for GDM.

The interface between taste and ingestion is coming to light in numerous ways through functional and clinical analyses at the cellular, systemic, and behavioral levels. This is especially the case for sweet taste, where the obvious connections with obesity, diabetes, and a host of other ills motivate a detailed examination of the influence of peptide hormones on taste.

\section{Acknowledgments}

Supported by NIH/NIDCD grant R01DC006021.

\section{References}

1. Kitagawa M, et al. Molecular genetic identification of a candidate receptor gene for sweet taste. Biochem Biophys Res Commun. 2001; 283:236-242. [PubMed: 11322794]

2. Max M, et al. Tas1r3, encoding a new candidate taste receptor, is allelic to the sweet responsiveness locus Sac. Nat Genet. 2001; 28:58-63. [PubMed: 11326277]

3. Montmayeur JP, et al. A candidate taste receptor gene near a sweet taste locus. Nat Neurosci. 2001; 4:492-498. [PubMed: 11319557]

4. Nelson G, et al. Mammalian sweet taste receptors. Cell. 2001; 106:381-390. [PubMed: 11509186] 
5. Lush IE. The genetics of tasting in mice. VI. Saccharin, acesulfame, dulcin and sucrose. Genet Res. 1989; 53:95-99. [PubMed: 2744455]

6. Reed DR. Gene mapping for taste related phenotypes in humans and mice. Appetite. 2000; 35:189190. [PubMed: 10986112]

7. Galindo-Cuspinera V, et al. A TAS1R receptor-based explanation of sweet 'water-taste'. Nature. 2006; 441:354-357. [PubMed: 16633339]

8. Jiang P, et al. The cysteine-rich region of T1R3 determines responses to intensely sweet proteins. J Biol Chem. 2004; 279:45068-45075. [PubMed: 15299024]

9. Nie Y, et al. Distinct contributions of T1R2 and T1R3 taste receptor subunits to the detection of sweet stimuli. Curr Biol. 2005; 15:1948-1952. [PubMed: 16271873]

10. Zhang Y, et al. Coding of sweet, bitter, and umami tastes: different receptor cells sharing similar signaling pathways. Cell. 2003; 112:293-301. [PubMed: 12581520]

11. Shin YK, et al. Modulation of taste sensitivity by GLP-1 signaling. J Neurochem. 2008; 106:455463. [PubMed: 18397368]

12. Martin B, et al. Modulation of taste sensitivity by GLP-1 signaling in taste buds. Ann N Y Acad Sci International Symposium on Olfaction and Taste. 2009; 1170:98-101.

13. Kawai K, et al. Leptin as a modulator of sweet taste sensitivities in mice. Proc Natl Acad Sci USA. 2000; 97:11044-11049. [PubMed: 10995460]

14. Shigemura N, et al. Leptin modulates behavioral responses to sweet substances by influencing peripheral taste structures. Endocrinology. 2004; 145:839-847. [PubMed: 14592964]

15. Sanematsu K, et al. Modulation and transmission of sweet taste information for energy homeostasis. Ann N Y Acad Sci International Symposium on Olfaction and Taste. 2009; 1170:102-106.

16. Accolla R, et al. Differential spatial representation of taste modalities in the rat gustatory cortex. $\mathrm{J}$ Neurosci. 2007; 27:1396-1404. [PubMed: 17287514]

17. Accolla R, Carleton A. Internal body state influences topographical plasticity of sensory representations in the rat gustatory cortex. Proc Natl Acad Sci USA. 2008; 105:4010-4015. [PubMed: 18305172] 